\title{
Prognostic factors for spinal chordomas and chondrosarcomas treated with postoperative pencil-beam scanning proton therapy: a large, single-institution experience
}

\author{
Fritz R. Murray, MD, James W. Snider, MD, ${ }^{2}$ Ralf A. Schneider, MD, ${ }^{1}$ Marc Walser, MD, ${ }^{1}$ \\ Alessandra Bolsi, MSc, ${ }^{1}$ Alessia Pica, MD, ${ }^{1}$ Antony J. Lomax, PhD, ${ }^{1,3}$ and Damien C. Weber, MD ${ }^{1,4,5}$ \\ ${ }^{1}$ Center for Proton Therapy, Paul Scherrer Institute, Villigen; ${ }^{3}$ Department of Physics, ETH, Zurich; ${ }^{4}$ Radiation Oncology \\ Department, University Hospital of Bern; ${ }^{5}$ Radiation Oncology Department, University Hospital of Zurich, Switzerland; and \\ 2Department of Radiation Oncology, University of Maryland School of Medicine, Baltimore, Maryland
}

\begin{abstract}
OBJECTIVE The aim of this paper was to evaluate the prognostic factors in surgical and adjuvant care for spinal chordomas and chondrosarcomas after surgery followed by high-dose pencil-beam scanning proton therapy (PBS-PT). METHODS From 1997 to 2016, 155 patients (61 female patients; median age 55 years) with spinal (cervical, $n=61$; thoracic, $n=29$; lumbar, $n=13$; sacral, $n=46$; pelvic, $n=6)$ classic chordomas $(n=116)$ and chondrosarcomas $(n=39$; most were low grade) were treated with maximal safe resection followed by PBS-PT (median dose prescribed: 74 Gy [relative biological effectiveness], range 48.6-77 Gy). The majority of patients $(n=153,98.7 \%)$ had undergone at least 1 resection prior to PBS-PT (median 1, range $0-5$; biopsy only, $n=2$ ). Fewer than half $(45.1 \%)$ of the surgeries were rated as gross-total resections (GTRs) prior to PBS-PT. Surgical stabilization (SS) was present in $39 \%$ of all patients $(n=60)$. Ninety-one patients (59\%) presented with macroscopic tumor at the start of PBS-PT. The median follow-up duration was 64.7 months (range 12.2-204.8 months).
\end{abstract}

RESULTS The 5-year local tumor control, disease-free survival (DFS), and overall survival were $64.9 \%(95 \% \mathrm{Cl} 56.3 \%$ $73.5 \%$ ), 59.4\% (95\% Cl 50.6\%-68.2\%), and 77.9\% (95\% Cl 70.6\%-85.2\%), respectively. In total, 63 patients ( $40.6 \%)$ experienced failure during the follow-up period: local only in $32(20.6 \%)$, distal only in $7(4.5 \%)$, local + distal in $19(12.3 \%)$, surgical pathway failure (SPF) only in $2(1.3 \%)$, local + SPF in $2(1.3 \%)$, and distal + SPF in $1(<1 \%)$. Univariate analysis identified gross residual disease, the presence of SS, and treatment era prior to 2008 as highly significant for worse outcome, with all 3 remaining significant on multivariate analysis. The type of surgery (GTR or subtotal resection/biopsy) and whether GTR was achieved by en bloc or curettage did not show a significant prognostic effect. Surgical complications prior to PBS-PT were present in $42.5 \%$ of all surgically treated patients and were seen more commonly in patients with multiple surgical interventions $(p=0.005)$ and those operated on with the intent of en bloc resection $(p=0.006)$.

CONCLUSIONS The extent of resection and metallic stabilization substantially influenced clinical outcomes for patients with spinal chordoma or chondrosarcoma despite high-dose adjuvant PBS-PT. Optimal upfront surgical management of these tumors continues to include GTR, as possible, with prompt adjuvant proton therapy.

https://thejns.org/doi/abs/10.3171/2019.11.SPINE1927

KEYWORDS spinal chordoma; spinal chondrosarcoma; pencil-beam scanning; proton therapy; prognostic factors; surgical resection; oncology

ABBREVIATIONS CTCAE = Common Terminology Criteria for Adverse Events; DFS = disease-free survival; GTR = gross-total resection; IMPT = intensity-modulated proton therapy; LC = local control; OS = overall survival; PBS-PT = pencil-beam scanning proton therapy; PSI = Paul Scherrer Institute; PTV = planning treatment volume; PTV1 = PTV subclinical dose; PTV2 = PTV prescription dose; RBE = relative biological effectiveness; SPF = surgical pathway failure; SS = surgical stabilization. SUBMITTED January 7, 2019. ACCEPTED November 15, 2019 INCLUDE WHEN CITING Published online January 31, 2020; DOI: 10.3171/2019.11.SPINE1927. 
$\mathrm{S}$ PINAL chordomas and chondrosarcomas present particularly challenging scenarios for clinicians in balancing maximization of disease control with minimization of treatment-associated morbidity. As a result of these tumors' juxtapositions, wide en bloc resection is rarely achievable without substantial long-term toxicity. Proximity to critical structures, especially the spinal cord, also challenges the delivery of the high doses of radiotherapy required to affect outcomes in these relatively radioresistant tumor subtypes.

With the advent of particle therapy, and especially pencil-beam scanning proton therapy (PBS-PT), long-term control of this disease and survival have substantially improved. $4,8,10,12,13,19,20,23$ To date, surgical approaches have varied, and multiple series have presented conflicting data regarding the importance of en bloc approaches or grosstotal resections (GTRs) for disease outcomes. $3,6,20,23,25,30$ In addition, because of the rarity of these tumors, most efforts have spanned multiple decades, extending across eras that saw multiple improvements in imaging, surgical, and radiotherapeutic practice.

At the Paul Scherrer Institute (PSI), we have delivered exclusively PBS-PT over the last 20 years, with the most frequently treated tumor histologies including chordomas and chondrosarcomas of the base of the skul127,28 and spine. ${ }^{23}$ We have retrospectively reviewed these patient outcomes and treatment-associated toxicities. Here, we detail our experience with spinal chordomas/chondrosarcomas treated with maximal safe resection and adjuvant proton radiotherapy. A particular focus of this effort was to assess surgical factors associated with resection of spinal chordomas and chondrosarcomas and their prognostic value for outcomes and toxicities associated with treatment of these tumors.

\section{Methods \\ Patients}

Between 1997 and 2016, 155 patients with spinal chordomas $(n=116)$ or chondrosarcomas $(n=39)$ were treated at PSI with PBS-PT. Of note, there were no de-differentiated chordomas, and only a small portion of chondrosarcomas $(n=3,7.7 \%)$ were high-grade tumors. All other tumors were described in the initial pathology report as classic chordomas and low-grade chondrosarcomas. No central pathology review, however, was undertaken. A retrospective review was undertaken on an ethics committee-approved protocol. Patients were included if they were at least 18 years old with a minimum follow-up of 12 months. They must also have received PBS-PT for a chordoma or chondrosarcoma centered below the clivus and along the spinal axis. Pathologic review to confirm histology was commonly but not uniformly performed at local institutions.

The median patient age was 55 years (range 23-82 years), with a slight male predominance (94 men and 61 women; Table 1). Spinal level was approximated by the center of tumor growth or postoperative bed. Lesions were more common in the cervical $(n=61)$ and sacral $(n=46)$ regions, rather than the thoracic $(\mathrm{n}=29)$ and lumbar $(\mathrm{n}$ $=13)$ spine. Although most patients $(n=109,70 \%)$ were treated as part of their initial course of therapy, 30\% (n $=46$ ) received PBS-PT during treatment for disease recurrence. Due to a systematic enlargement of treatment volumes in 2008 after making an unpublished interim analysis, this series was stratified prior to analysis into patients treated before $2008(n=64,41 \%)$ and those treated in 2008 and later $(\mathrm{n}=91,59 \%)$.

A total of 153 patients underwent resection prior to radiotherapy. Two patients underwent biopsy only. Of these, 69 underwent GTR as defined intraoperatively. Twentyseven (39\%) GTRs were performed by an en bloc technique, as defined by the primary surgeon in associated operative notes, whereas $38(55 \%)$ procedures were curettage. Three resections were unable to be characterized with regard to technique, and 1 diagnosis was made by an incidental histological finding during intervention for a benign condition. Of note, the majority of patients $(\mathrm{n}=91,59 \%)$ had appreciable gross disease on pre-PBS-PT simulation imaging.

Sixty (39\%) patients (44 with chordoma and 16 with chondrosarcoma) had surgical stabilization (SS) in place at the time of PBS-PT delivery.

\section{Radiation Therapy}

Immobilization and planning techniques have been previously described elsewhere. ${ }^{23}$ Patients were most often treated with a 2-phase technique delivering 54 Gy (relative biological effectiveness [RBE]) to a larger volume to address subclinical disease, followed by a boost to a total of 70-74 Gy (RBE) (range 48.6-77.0 Gy [RBE]) to the immediate postoperative bed and any gross residual disease. Treatment generally employed $2-4$ posterior beams and usually the first plan was single-field uniform-dose treatment with no organ-at-risk sparing..$^{14}$ The treatment plan was changed to intensity-modulated proton therapy (IMPT) after 30-36 Gy (RBE). Standardized institutional dose constraints have been previously described. ${ }^{24}$

\section{Follow-Up}

The majority of patients were followed closely with MRI after treatment at 6 weeks and every 6 months thereafter. At PSI, posttreatment imaging and outside physician records were and continue to be reviewed regularly by the entire clinical team to characterize disease status as well as toxicity. Physician notes, summaries from these conferences, posttreatment imaging, and external records obtained by the research team were retrospectively reviewed for this analysis. Surgical complications were defined as those directly attributable to resection intervention and those arising postoperatively, prior to initiation of radiotherapy. However, based on the inability to clarify which complications were expected by the surgeon prior to each individual intervention, certain "expected toxicities" were likely included as "complications" in the underlying analysis. Adverse events were graded based on the US National Institutes of Health Common Terminology Criteria for Adverse Events (CTCAE) grading system (v4.0).

\section{Outcomes and Statistical Analysis}

Local control (LC), disease-free survival (DFS), and 
TABLE 1. Patient and treatment characteristics

\begin{tabular}{|c|c|c|c|}
\hline Characteristic & $\begin{array}{l}\text { All Patients } \\
(n=155)\end{array}$ & $\begin{array}{l}\text { Chordoma } \\
(n=116)\end{array}$ & $\begin{array}{l}\text { Chondrosarcoma } \\
\qquad(\mathrm{n}=39)\end{array}$ \\
\hline \multicolumn{4}{|l|}{ Sex, n (\%) } \\
\hline Male & $94(61)$ & $69(60)$ & $25(64)$ \\
\hline Female & $61(39)$ & $47(41)$ & $14(63)$ \\
\hline \multicolumn{4}{|l|}{ Age in yrs } \\
\hline Median & 55 & 57 & 50 \\
\hline Range & $23-82$ & $25-82$ & $23-78$ \\
\hline \multicolumn{4}{|l|}{ Histology \& type, n (\%) } \\
\hline Chordoma & $116(75)$ & & \\
\hline Classic & & $116(100)$ & \\
\hline De-differentiated & & $0(0)$ & \\
\hline Chondrosarcoma & $39(25)$ & & \\
\hline Low grade (grade I-II) & & & $36(92)$ \\
\hline High grade (grade III) & & & $3(8)$ \\
\hline \multicolumn{4}{|l|}{ Tumor site/spinal level, n (\%) } \\
\hline Cervical & $61(39)$ & $50(43)$ & $11(28)$ \\
\hline Thoracic & $29(19)$ & $8(7)$ & $21(54)$ \\
\hline Lumbar & $13(8)$ & $13(11)$ & $0(0)$ \\
\hline Sacral & $46(30)$ & $45(39)$ & $1(3)$ \\
\hline Pelvic & $6(4)$ & $0(0)$ & $6(15)$ \\
\hline \multicolumn{4}{|l|}{ Timing of PBS-PT, n (\%) } \\
\hline Initial diagnosis & $109(70)$ & $84(72)$ & $25(64)$ \\
\hline Recurrence & $46(30)$ & $32(28)$ & $14(26)$ \\
\hline \multicolumn{4}{|l|}{ Dose in Gy (RBE) } \\
\hline Median & 74 & 74 & 70 \\
\hline Range & $48.6-77$ & $59.4-77$ & $48.6-75.8$ \\
\hline \multicolumn{4}{|l|}{ Radiation modality, n (\%) } \\
\hline Proton only & $137(88)$ & $104(90)$ & $133(85)$ \\
\hline Combined photon-proton & $18(12)$ & $12(10)$ & $6(15)$ \\
\hline \multicolumn{4}{|l|}{ Era, $\mathrm{n}(\%)$} \\
\hline Pre-2008 & $64(41)$ & $44(38)$ & $20(51)$ \\
\hline 2008 \& later & $91(59)$ & $72(62)$ & $19(49)$ \\
\hline \multicolumn{4}{|l|}{ Resection, $\mathrm{n}(\%)$} \\
\hline Yes & $153(99)$ & $115(99)$ & $38(97)$ \\
\hline GTR at 1 st resection & $69(45)$ & $53(47)$ & $16(42)$ \\
\hline GTR at last resection & $71(46)$ & $57(50)$ & $14(37)$ \\
\hline \multicolumn{4}{|c|}{ If 1st surgery GTR: technique, $n(\%)^{*}$} \\
\hline En bloc & $27(39)$ & $23(43)$ & $4(25)$ \\
\hline Intralesional & $38(55)$ & $29(55)$ & $9(56)$ \\
\hline \multicolumn{4}{|c|}{ Macroscopic tumor prior to PBS-PT, n (\%) } \\
\hline Present & $91(59)$ & $66(57)$ & $25(64)$ \\
\hline Absent & $64(41)$ & $50(43)$ & $14(36)$ \\
\hline SS present, n (\%) & $60(39)$ & $44(43)$ & $16(41)$ \\
\hline \multicolumn{4}{|l|}{ PTV1 in $\mathrm{cm}^{3}$} \\
\hline Median & 599 & 809 & 386 \\
\hline Range & $28-4977$ & $42-4024$ & $28-4977$ \\
\hline Median pre-2008 & 523 & 645 & 332 \\
\hline Median 2008 \& later & 866 & 897 & 395 \\
\hline
\end{tabular}


» CONTINUED FROM PAGE 923

TABLE 1. Patient and treatment characteristics

\begin{tabular}{lllc}
\hline \multicolumn{1}{c}{ Characteristic } & $\begin{array}{c}\text { All Patients } \\
(\mathrm{n}=155)\end{array}$ & $\begin{array}{c}\text { Chordoma } \\
(\mathrm{n}=116)\end{array}$ & $\begin{array}{c}\text { Chondrosarcoma } \\
(\mathrm{n}=39)\end{array}$ \\
\hline PTV2 $\mathrm{in} \mathrm{cm}^{3}$ & & & \\
\hline Median & 368 & 415 & 259 \\
\hline Range & $11-3749$ & $25-3749$ & $11-3076$ \\
\hline Median pre-2008 & 327 & 345 & 212 \\
\hline Median 2008 \& later & 393 & 445 & 268 \\
\hline * & & & \\
\hline
\end{tabular}

for a traumatic vertebral fracture with an incidental histopathological malignancy.

overall survival (OS) were assessed as the primary disease-related endpoints. Local failure was declared if imaging or clinical progression or recurrence was noted on the date of first suggestion of recurrence. Disease progression was notated if there was local, regional, or distal recurrence. OS events were defined per date of confirmed death. LC, DFS, and OS were censored at the date of last follow-up if no event had occurred.

Statistical analysis was performed using the IBM SPSS Statistics software package (IBM Corp.).

\section{Results}

Patient characteristics and follow-up observations are displayed in Tables 1 and 2, respectively. All 155 patients were followed up for a median of 64.7 months (range 12.2-204.8 months). After stratification by treatment era, the median follow-up for patients treated prior to or during 2007 ( $n=64$ ) was 90.6 months (range 17.1-204.8 months), and for patients treated after $2007(\mathrm{n}=91)$ it was 57 months (range 12.2-110.7 months). Tumor recurrence or progression was observed in $63(40.6 \%)$ patients (45 [38.8\%] with chordoma; 18 [46.2\%] with chondrosarcoma): local only in $32(20.6 \%)$, distal only in $7(4.5 \%)$, local + distal in 19 $(12.3 \%)$, surgical pathway failure (SPF) only in $2(1.3 \%)$, local + SPF in $2(1.3 \%)$, and distal + SPF in $1(<1 \%)$. A total of 47 patients died (tumor related, $\mathrm{n}=32$ [68.1\%]) during the follow-up period (32 [27.6\%] with chordoma; 15 [38.5\%] with chondrosarcoma). Of note, at the start of PBS-PT, 91 patients (59\%) had macroscopic disease, and $60(39 \%)$ had undergone SS with a metallic implant. The 5 -year LC, DFS, and OS for the entire cohort (Tables 3 and 4) were $64.9 \%$ (95\% CI 56.3\%-73.5\%), 59.4\% (95\% CI $50.6 \%-68.2 \%$ ), and $77.9 \%$ (95\% CI 70.6\%-85.2\%). LC rates at 5 years for chordoma and chondrosarcoma were $67.9 \%$ (95\% CI $58.1 \%-77.7 \%$ ) and $55.9 \%$ (95\% CI $38.3 \%-$ $73.5 \%$ ), respectively.

Univariate analysis did not show a significant difference between the two histological groups with regard to LC ( $p$ $=0.49$ ). In accordance with this, 5-year DFS (chordoma: 62.1\%, 95\% CI 52.1\%-72.1\%; chondrosarcoma: $51.7 \%$, 95\% CI 34.3\%-69.1\%) and 5-year OS (chordoma: 81.6\%, 95\% CI 73.6\%-89.6\%; chondrosarcoma: $67.3 \%$, 95\% CI $51.2 \%-83.4 \%$ ) were also not substantially different ( $\mathrm{p}=$ 0.55 and $p=0.36$ for DFS and OS, respectively).

As a result, univariate and multivariate analyses (Ta- bles 5 and 6) for LC, DFS, and OS, based on key surgical and adjuvant therapy prognostic factors, were performed for the entire cohort with these histologies combined. After stratification for treatment era, analysis of the treated volumes showed a significant increase in size of planning treatment volumes (PTVs) for patients treated after 2007 (increase of the median PTV subclinical dose [PTV1] by $40 \%, \mathrm{p}=0.01)$. The median PTV prescription dose (PTV2) increased by $18 \%$ but was not statistically significantly different from that in the prior era $(p=0.29)$. Of note, the distribution of tumors throughout the spine before and after 2008 remained relatively balanced (cervical, thoracic, lumbar, sacral, and pelvic, pre-2008: 25, 12, 9, 16, and 2, respectively; 2008 and later: 36, 17, 4, 30, and 4, respectively).

\section{Local Control}

On univariate analysis (Table 5), the presence of metallic SS significantly worsened 5-year LC (with SS, 5-year $\mathrm{LC}=50.0 \%$; without SS, 5-year LC $=73.4 \% ; \mathrm{p}=0.02)$. In addition, the treatment era significantly influenced 5-year LC (pre-2008: 5-year LC $=52.1 \%$; 2008 and later: 5-year $\mathrm{LC}=77.0 \% ; \mathrm{p}<0.001)$. Furthermore, the total number of surgeries trended toward significance, with a higher number of surgeries prognosticating for worsened control rates (total number of surgeries $\leq 1,5$-year LC $=71.5 \%$; total number of surgeries $\geq 2,5$-year $L C=56.0 \% ; p=0.05$ ). A clear relationship was not evident between interval time between surgery and PBS-PT and LC in this setting, with no significant difference found in LC based on time (dichotomous at the median) from the first resection to the start of PBS-PT $(<7.4$ months, $p=0.09)$. On univariate analysis, no statistical significances for 5-year LC rates were found in the case of absent gross residual disease $(\mathrm{p}$ $=0.17)$ or spinal region $(p=0.73)$. The latter was categorized as either mobile spine (cervical, thoracic, and lumbar) or pelvic and sacral. On multivariate analysis (Table $6)$, later treatment era (2008 and later, $p=0.001)$, total number of surgeries $(\mathrm{p}=0.03)$, absence of SS $(\mathrm{p}=0.03)$, and absence of gross residual disease $(\mathrm{p}=0.033)$ were significant prognosticators for improved LC.

\section{Disease Control}

In total, 27 distal failures and 5 SPFs (new chordomas or chondrosarcomas arising well outside the current field 
TABLE 2. Follow-up observations*

\begin{tabular}{|c|c|}
\hline & No. of Events (\%) \\
\hline \multicolumn{2}{|l|}{ Survival (entire cohort) } \\
\hline Alive & $108(69.7)$ \\
\hline Dead & $47(30.3)$ \\
\hline Death, tumor related & $32(20.6)$ \\
\hline \multicolumn{2}{|l|}{ Local failure } \\
\hline Yes & $53(34.2)$ \\
\hline No & $102(65.8)$ \\
\hline Local failure only & $32(20.6)$ \\
\hline \multicolumn{2}{|l|}{ Distal failure } \\
\hline Yes & $27(17.4)$ \\
\hline No & $128(82.6)$ \\
\hline Distal failure only & $7(4.5)$ \\
\hline Distal + local failure & $19(12.3)$ \\
\hline \multicolumn{2}{|l|}{ SPF } \\
\hline Yes & $5(3.2)$ \\
\hline No & $150(96.7)$ \\
\hline SPF only & $2(1.3)$ \\
\hline SPF + local failure & $2(1.3)$ \\
\hline SPF + distal failure & $1(<1)$ \\
\hline \multicolumn{2}{|l|}{ Any failure } \\
\hline Yes & $63(40.6)$ \\
\hline No & $92(59.4)$ \\
\hline \multicolumn{2}{|c|}{ Late toxicity from proton therapy } \\
\hline Yes & $52(33.5)$ \\
\hline CTCAE grade $\geq 3$ & $12(7.7)$ \\
\hline \multicolumn{2}{|c|}{ Surgical complication before proton therapy } \\
\hline Yes & $65(42.5)$ \\
\hline No & $88(57.5)$ \\
\hline \multicolumn{2}{|l|}{ Chordoma } \\
\hline Local failure & $38(32.8)$ \\
\hline Distal failure & $20(17.2)$ \\
\hline SPF & $3(2.6)$ \\
\hline Any failure & $45(38.8)$ \\
\hline Dead at last FU & $32(27.6)$ \\
\hline \multicolumn{2}{|l|}{ Chondrosarcoma } \\
\hline Local failure & $15(38.5)$ \\
\hline Distal failure & $7(17.9)$ \\
\hline SPF & $2(5.1)$ \\
\hline Any failure & $18(46.2)$ \\
\hline Dead at last FU & $15(38.5)$ \\
\hline
\end{tabular}

FU = follow-up.

* Overall median follow-up: 64.7 months (range 12.2-204.8 months). Follow-up was stratified by treatment era. Pre-2008 median follow-up: 90.6 months (range 17.1-204.8 months); 2008 and later median follow-up: 57 months (range 12.2-110.7 months).

in the spine), along with the 53 local failures, were documented as disease failures (Table 2). Consistent with the findings for LC, univariate analysis identified a significant influence of SS $(\mathrm{p}=0.01)$ and pre-2008 treatment era ( $\mathrm{p}$ $=0.001$ ) on outcomes, both corresponding with worsened
TABLE 3. Subgroup results: LC, DFS, and OS

\begin{tabular}{ccc}
\hline Subgroup* & Rate & $95 \% \mathrm{Cl}$ \\
\hline 5-yr LC & & \\
\hline Entire cohort & 64.9 & $56.3-73.5 \%$ \\
\hline Chordoma & 67.9 & $58.1-77.7 \%$ \\
\hline Chondrosarcoma & 55.9 & $38.3-73.5 \%$ \\
\hline 5-yr DFS & & \\
\hline Entire cohort & 59.4 & $50.6-68.2 \%$ \\
\hline Chordoma & 62.1 & $52.1-72.1 \%$ \\
\hline Chondrosarcoma & 51.7 & $34.3-69.1 \%$ \\
\hline 5-yr OS & & \\
\hline Entire cohort & 77.9 & $70.6-85.2 \%$ \\
\hline Chordoma & 81.6 & $73.6-89.6 \%$ \\
\hline Chondrosarcoma & 67.3 & $51.2-83.4 \%$ \\
\hline
\end{tabular}

${ }^{*}$ Entire cohort $=$ both histologies included.

DFS (Table 5). In addition, the absence of gross residual disease trended toward significance $(\mathrm{p}=0.08)$. On multivariate analysis (Table 6), the absence of gross residual disease $(p=0.02)$ and treatment in the later era (2008 and later, $\mathrm{p}=0.001)$ significantly increased DFS. In addition, multivariate analysis showed a trend toward a worse outcome with the presence of SS $(p=0.06)$.

\section{Overall Survival}

On univariate analysis (Tables 5 and 6), treatment in the later era (pre-2008: 5-year OS $=65.6 \% ; 2008$ and later: 5 -year OS $=91.1 \% ; \mathrm{p}=0.001)$, absence of gross residual disease (gross residual: 5 -year $\mathrm{OS}=70.4 \%$; no gross residual: 5-year OS $=89.8 \% ; \mathrm{p}=0.001$ ), and absence of SS (SS: 5 -year OS $=66.8 \%$; no SS: 5-year OS = 84.8\%; $\mathrm{p}=0.002$ ) significantly correlated with improved OS. In addition, all of these factors proved to be significant prognosticators on multivariate analysis (treatment era, $p=0.002$; gross residual disease, $p<0.0001 ; S S, p=0.01$ ). In contrast to LC and DFS, on univariate analysis the total number of surgeries $(\mathrm{p}=0.03)$ and whether the type of first $(\mathrm{p}=0.02)$ and last $(p<0.001)$ resections were GTRs also showed statistical significance for prolonged survival. These factors did not hold significance on multivariate analysis. Furthermore, age older than 55 years at time of PBS-PT showed a trend toward worsened 5-year OS $(\mathrm{p}=0.06)$.

\section{Surgical Complications}

Surgical complications were encountered in $42.5 \%$ of all patients $(n=65)$ who underwent at least 1 resection. Neurological complications were present in 43 patients, including sphincter/bladder dysfunction in 14 and motor deficits in 22. Twelve patients experienced postoperative infections, and 6 patients experienced postoperative hemorrhage. On univariate analysis, an increasing number of surgeries $(\mathrm{p}=0.01)$ and en bloc-intention resections $(\mathrm{p}$ $=0.01$ ) were significantly correlated with increased rates of surgical complication. In addition, surgeries performed for recurrent disease trended toward significant prediction of higher rates of complication ( $\mathrm{p}=0.05$ ). To our knowl- 
TABLE 4. Results stratified by era, gross disease, and SS use

\begin{tabular}{|c|c|c|c|}
\hline & $\begin{array}{c}\text { No. of } \\
\text { Events (\%) }\end{array}$ & Rate & $95 \% \mathrm{Cl}$ \\
\hline \multicolumn{4}{|l|}{ Era } \\
\hline \multicolumn{4}{|c|}{ Pre-2008 $(n=64)$} \\
\hline 5-yr LC & $37(57.8)$ & 52.1 & $39.4-64.8 \%$ \\
\hline 5-yr DFS & $42(65.6)$ & 46.3 & $33.8-58.8 \%$ \\
\hline $5-y r$ OS & $36(60.9)$ & 65.6 & $54.0-77.2 \%$ \\
\hline \multicolumn{4}{|c|}{2008 \& later $(n=91)$} \\
\hline 5-yr LC & $16(17.6)$ & 77.0 & $66.4-87.6 \%$ \\
\hline 5-yr DFS & $21(32.8)$ & 71.6 & $60.2-83.0 \%$ \\
\hline $5-y r$ OS & $8(8.8)$ & 91.1 & $84.2-98.0 \%$ \\
\hline \multicolumn{4}{|l|}{ Gross disease } \\
\hline \multicolumn{4}{|l|}{ Yes (n= 91) } \\
\hline 5-yr LC & $34(37.4)$ & 59.6 & $48.2-71.0 \%$ \\
\hline 5-yr DFS & $41(45.1)$ & 52.6 & $41.2-64.0 \%$ \\
\hline $5-y r$ OS & $33(36.6)$ & 70.4 & $60.2-80.6 \%$ \\
\hline \multicolumn{4}{|l|}{ No $(n=64)$} \\
\hline 5-yr LC & $19(29.7)$ & 72.9 & $60.2-85.6 \%$ \\
\hline 5-yr DFS & $22(34.4)$ & 69.5 & $56.0-83.0 \%$ \\
\hline 5 -yr OS & $14(21.8)$ & 89.8 & $81.4-98.2 \%$ \\
\hline \multicolumn{4}{|l|}{ SS use } \\
\hline \multicolumn{4}{|c|}{ Implant $(n=60)$} \\
\hline 5-yr LC & $26(43.3)$ & 50.0 & $34.9-65.1 \%$ \\
\hline 5-yr DFS & $30(50.0)$ & 43.9 & $29.0-58.8 \%$ \\
\hline $5-y r$ OS & $29(48.3)$ & 66.8 & $53.5-80.1 \%$ \\
\hline \multicolumn{4}{|c|}{ No implant $(n=95)$} \\
\hline 5-yr LC & $27(28.4)$ & 73.4 & $63.4-83.4 \%$ \\
\hline 5-yr DFS & $33(34.8)$ & 67.9 & $57.5-78.3 \%$ \\
\hline $5-y r$ OS & $18(18.9)$ & 84.8 & $76.8-92.8 \%$ \\
\hline
\end{tabular}

edge, no hardware failures were encountered in this series, although the retrospective nature and the outside hospital surgical interventions make this difficult to definitively clarify.

\section{Radiation-Induced Toxicities}

Long-term radiation-induced toxicities were encountered in $33.5 \%$ of all patients $(n=52)$. A total of 12 patients (7.7\%) presented with high-grade toxicities ( $\geq$ grade 3 ). Ten patients had grade 3 toxicities, including esophageal strictures requiring dilatation $(n=2)$, insufficiency fractures $(n=3)$, soft-tissue necrosis $(n=1)$, subcutaneous fistula $(n=1)$, neuropathic pain $(n=1)$, femoral insufficiency requiring hip replacement $(\mathrm{n}=1)$, and ureteral stenosis (n $=1$ ). Another 2 patients presented with grade 4 toxicities: myelitis causing quadriplegia in a patient presenting with quadriplegia from preoperative spinal cord compression that initially improved after therapy and then worsened, and a laryngeal necrosis requiring hyperbaric oxygen therapy. One patient, who received a combined photon-proton treatment for sacral chordoma and was already included in the group of 10 with high-grade toxicities for a subcutaneous fistula, died 48 months after completion of therapy due to metastatic rhabdomyosarcoma of the bladder, deemed to be potentially radiation induced. Of note, the 19.8-Gy (RBE) proton therapy boost contributed a negligible dose to the bladder in this case.

\section{Discussion}

The relative radio-insensitivity of chordomas and chondrosarcomas and their proximity to critical organs at risk when arising around the spinal axis make these tumors particularly challenging for clinicians. Although radical surgery is often not feasible, maximal safe resection followed by appropriate adjuvant therapy remains the standard of care. ${ }^{7,9,31}$ Multiple series have demonstrated that high rates of LC, DFS, and OS are achievable for extracranial chordomas and chondrosarcomas, but that thorough maximal safe resection and high doses of radiotherapy (> 70 Gy [RBE]) are required. ${ }^{10,15,18,20}$ Particle therapy offers conformality and dose that is superior to traditional photon techniques, allowing a dose escalation. The results for LC, DFS, and OS in the current study (the largest reported thus far with exclusively PBS-PT) are consistent with results published by other particle therapy centers. ${ }^{11,12,20}$

The importance of the surgeon's skill and experience in approaching spinal chordoma and chondrosarcoma cannot be overstated. Several series have documented the importance of achieving maximal resection without unacceptable patient morbidity. These efforts have variably determined that either the extent of resection or surgical approach (en bloc vs curettage) may be prognostic for disease outcomes. For example, Rotondo et al., Bergh et al., Boriani et al., and Talac et al. each determined that en bloc resection techniques reduced rates of recurrence., $3,6,20,25$ Conversely, Snider et al. and York et al. emphasized the importance of rendering the patient free of gross disease prior to the initiation of adjuvant therapy. ${ }^{23,30}$ Schwab et al. noted that, although curettage resections were associated with a higher chance of local failure, the administration of adjuvant radiotherapy seemed to reduce the importance of wide versus "contaminated" margins. ${ }^{22}$

In our series, GTR was of prognostic value in predicting outcomes by multivariate analysis. Neither univariate nor multivariate analysis showed statistical significance when comparing en bloc and curettage surgical approaches for disease outcomes of LC, DFS, and OS ( $p=0.32, p$ $=0.47$, and $p=0.53$, respectively). A particular strength of this analysis is not only its use of modern particle therapy (PBS-PT) but also its use of modern diagnostic imaging (MR) in the radiotherapy simulation and follow-up processes to define disease/recurrence. MRI may have defined residual disease that could have been underappreciated in previous series despite "en bloc" techniques. However, because of this study's retrospective nature and the referral pattern at PSI, the surgical approach was documented by review of surgical notes from various surgeons at numerous institutions from multiple countries. Variability in reporting could clearly have affected results.

In addition, we included a detailed univariate analysis of factors predictive of significant posttreatment morbidity. Resection of spinal tumors is generally associated with high rates of morbidity, ranging from $35 \%$ to $46 \% .5,7,16$ 
TABLE 5. Univariate analysis: LC, DFS, and OS

\begin{tabular}{|c|c|c|c|c|}
\hline \multirow[b]{2}{*}{ Characteristic } & \multirow[b]{2}{*}{ Value } & \multicolumn{3}{|c|}{$\mathrm{p}$ Value } \\
\hline & & LC & DFS & OS \\
\hline Age, yrs & & 0.485 & 0.328 & 0.058 \\
\hline Median & 55 & & & \\
\hline Range & $23-82$ & & & \\
\hline Sex & & 0.921 & 0.497 & 0.604 \\
\hline Female & $61(39)$ & & & \\
\hline Male & $94(61)$ & & & \\
\hline Total dose, Gy & & 0.169 & 0.398 & 0.073 \\
\hline$<70$ & $23(15)$ & & & \\
\hline$\geq 70$ & $132(85)$ & & & \\
\hline Modality & & 0.718 & 0.987 & 0.844 \\
\hline Proton only & $137(88)$ & & & \\
\hline Photon-PBS-PT combined & $18(12)$ & & & \\
\hline Spine region & & 0.729 & 0.548 & 0.576 \\
\hline Cervical, lumbar, \& thoracic & $103(66)$ & & & \\
\hline Pelvic sacral & $52(34)$ & & & \\
\hline Treatment setting & & 0.358 & 0.542 & 0.226 \\
\hline Initial & $109(70)$ & & & \\
\hline Recurrence & $46(30)$ & & & \\
\hline Gross residual tumor & & 0.170 & 0.076 & 0.001 \\
\hline Yes & $91(59)$ & & & \\
\hline No & $64(41)$ & & & \\
\hline SS & & 0.022 & 0.014 & 0.002 \\
\hline Yes & $60(39)$ & & & \\
\hline No & $95(61)$ & & & \\
\hline Treatment era & & 0.001 & 0.001 & 0.001 \\
\hline Pre-2008 (focal) & $64(41)$ & & & \\
\hline 2008 \& later (comprehensive) & $91(59)$ & & & \\
\hline No. of surgeries & & 0.053 & 0.122 & 0.033 \\
\hline $0-1$ & $90(58)$ & & & \\
\hline$\geq 2$ & $65(42)$ & & & \\
\hline Type of 1 st surgery* & & 0.761 & 0.382 & 0.023 \\
\hline GTR & $69(45)$ & & & \\
\hline STR/biopsy & $84(55)$ & & & \\
\hline Technique if 1 st surgery GTR† & & 0.317 & 0.474 & 0.527 \\
\hline En bloc & $27(39)$ & & & \\
\hline Curettage & $38(55)$ & & & \\
\hline PTV1 in $\mathrm{cm}^{3}$ & & 0.812 & 0.715 & 0.529 \\
\hline Median & 599 & & & \\
\hline Range & $28-4977$ & & & \\
\hline PTV2 in $\mathrm{cm}^{3}$ & & 0.581 & 0.514 & 0.574 \\
\hline Median & 368 & & & \\
\hline Range & $11-3749$ & & & \\
\hline
\end{tabular}

STR = subtotal resection.

Values represent the number of patients (\%) unless stated otherwise.

* Type of first surgery unknown in 2 patients.

† Of all patients who underwent GTR, the resection technique of 3 patients was unknown. One patient underwent surgery for a traumatic vertebral fracture with an incidental histopathologic finding. 
TABLE 6. Multivariate analysis: LC, DFS, and OS

\begin{tabular}{cc}
\hline Endpoint \& Characteristic $^{*}$ & p Value \\
\hline LC & \\
\hline Gross residual disease & 0.033 \\
\hline SS & 0.028 \\
\hline Era (pre-2008/2008 \& later) & 0.001 \\
\hline Total no. of surgeries & 0.026 \\
\hline DFS & \\
\hline Gross residual disease & 0.018 \\
\hline SS & 0.055 \\
\hline Era (pre-2008/2008 \& later) & 0.001 \\
\hline OS & $<0.0001$ \\
\hline Gross residual disease & 0.011 \\
\hline SS & 0.002 \\
\hline Era (pre-2008/2008 \& later)
\end{tabular}

* Gross residual disease, SS, the pre-2008 era, and a growing total number of surgeries prognosticate for worsened outcomes.

Wei et al. reported postoperative complications as high as $74 \% .{ }^{29}$ The morbidity associated with resection for sacral tumors might be even higher, with published rates of complications up to $100 \% .22,26$ In our series, we similarly documented surgical complications in $42.5 \%$ of all operated patients, with the majority $(66 \%)$ being of a neurological nature. Toxicity rates were, not surprisingly, associated with multiple surgical interventions and recurrent disease (Table 5). Increasing clinical target volume size, which corresponds to the extent of resection and resultant surgical bed, was significantly associated with the surgical complication rate.

En bloc approaches were also associated with higher complication rates in this series. Amendola et al. previously published the results of 20 years of experience in a single institution, emphasizing en bloc resection. ${ }^{2}$ The surgical complication rate was $41.7 \%$, with a $1.9 \%$ surgical mortality rate. They argued that the high morbidity rate associated with en bloc resections, including resultant functional limitations, can be justified by the long-term survival afforded, especially in the era of high-dose adjuvant particle therapy. ${ }^{10,15,18,20}$ Consistent with this finding, $77.9 \%$ of patients included in our series were alive at 5 years. Although surgical complication rates were relatively high, the rate of radiation-induced high-grade (CTCAE events grade $\geq 3$ ) toxicity was less than $8 \%$.

In our series, we observed that more than half of the treatment failures (32/63 [50.8\%]; Table 2) were local only. This is in line with the spinal chordoma series from Boston that reported on the outcome of 126 patients treated with surgery and high-dose (median 72.4 Gy [RBE]) proton therapy. ${ }^{20}$ After a median follow-up of 41 months, the estimated OS and locoregional control was remarkably similar to ours, namely, $81 \%$ and $62 \%$, respectively. Noteworthy, these authors observed a significantly higher rate of LC and locoregional control for primary tumors treated with preoperative low-dose radiation therapy. Our institution does not routinely treat spinal chordoma patients with neo-adjuvant radiation, and it remains to be demonstrated if a preoperative proton therapy is advisable in this setting. More data stemming from ideally prospective studies are needed to answer this critical question.

These findings have informed and support our current clinical approach: patients with spinal chordoma or chondrosarcoma substantially benefit from a planned, multidisciplinary approach in high-volume centers. We emphasize the importance of maximal safe resection (GTR) on first intervention, regardless of technique, and recommend high-dose adjuvant proton therapy promptly at initial presentation rather than observing for recurrence, regardless of the extent of resection. Of note, we observed a limited number of surgical pathway recurrences $(\mathrm{n}=2,1.3 \%)$ only (Table 2). This is probably consequential to the fact that our institution prophylactically treated the surgical pathway to a dose of $54 \mathrm{~Gy}$ (RBE). In particular, early adjuvant radiation in order to prevent salvage treatment, which has been linked to poorer outcomes, has been supported by earlier reviews., ${ }^{1,17}$

Metallic SS was, again, highly prognostic for worsened LC, DFS, and OS. This finding is consistent with previous reports from our institution. ${ }^{21,23}$ Concern initially focused on the possibility that titanium materials might be contributing to "shadowing" or under-dosage to disease in delivery of adjuvant particle therapy. However, the consistency of this series' outcomes with previous efforts combining photon and proton techniques to similar total doses $(\geq 70$ Gy [RBE]) is reassuring that this is likely not the case. In addition, a pattern of failure analysis reported previously does not seem to indicate that local recurrences correlate in position and geometry with surgical SS or expected resultant dose shadowing. ${ }^{23} \mathrm{SS}$ is generally required in the setting of more extensive initial disease, and may only, therefore, be representative of worse initial disease, which carries a worse prognosis. Nevertheless, the use of alternative implant materials, such as carbon-reinforced polyetheretherketone, may result in improved tumor and target visualization, less CT-artifact delineation times, more accurate proton dosimetry, and more reliable follow-up imaging. This technological enhancement could be helpful in improving outcomes for patients with spinal chordoma and chondrosarcoma going forward.

Treatment era was also highly prognostic in this series. After an internal review of the failures of the early treatment era (prior to 2008), the PSI physician group decided to systematically enlarge treatment volumes. In retrospect, we found a significant enlargement of PTV1 and PTV2 (increase in median volume by $40 \%$ and $18 \%$, respectively), which significantly improved long-term results. However, this result could be confounded by other improvements in treatment planning, surgical techniques, and referral patterns across the two eras.

This analysis, despite inherent limitations as a retrospective study, represents one of the largest cohorts of patients with spinal chordoma and chondrosarcoma reported to date. With long-term follow-up of patients treated with adjuvant PBS-PT at a single institution, we believe that this study is particularly informative to the community in improving outcomes for these clinically challenging diseases. This effort is unique in its focus on surgical prognostic factors for both functional and oncological outcomes. 


\section{Conclusions}

Resection (with the goal of GTR) in experienced hands remains of utmost importance in the management of spinal chordoma and chondrosarcoma. However, en bloc resections might not be superior to curettage techniques, and more aggressive, wide approaches are likely associated with significantly higher rates of surgical complications and functional morbidity. In the era of high-dose adjuvant proton therapy, which has yielded substantially improved treatment outcomes, this is of particular importance, because patients may live for many years with the morbidities associated with not only their disease but also their therapy.

\section{References}

1. Ailon T, Torabi R, Fisher CG, Rhines LD, Clarke MJ, Bettegowda C, et al: Management of locally recurrent chordoma of the mobile spine and sacrum: a systematic review. Spine (Phila Pa 1976) 41 (Suppl 20):S193-S198, 2016

2. Amendola L, Cappuccio M, De Iure F, Bandiera S, Gasbarrini A, Boriani S: En bloc resections for primary spinal tumors in 20 years of experience: effectiveness and safety. Spine J 14:2608-2617, 2014

3. Bergh P, Gunterberg B, Meis-Kindblom JM, Kindblom LG: Prognostic factors and outcome of pelvic, sacral, and spinal chondrosarcomas: a center-based study of 69 cases. Cancer 91:1201-1212, 2001

4. Bjornsson J, Wold LE, Ebersold MJ, Laws ER: Chordoma of the mobile spine. A clinicopathologic analysis of 40 patients. Cancer 71:735-740, 1993

5. Boriani S, Bandiera S, Donthineni R, Amendola L, Cappuccio M, De Iure F, et al: Morbidity of en bloc resections in the spine. Eur Spine J 19:231-241, 2010

6. Boriani S, De Iure F, Bandiera S, Campanacci L, Biagini R, Di Fiore M, et al: Chondrosarcoma of the mobile spine: report on 22 cases. Spine (Phila Pa 1976) 25:804-812, 2000

7. Boriani S, Gasbarrini A, Bandiera S, Ghermandi R, Lador R: En bloc resections in the spine: the experience of 220 patients during 25 years. World Neurosurg 98:217-229, 2017

8. Cummings BJ, Hodson DI, Bush RS: Chordoma: the results of megavoltage radiation therapy. Int J Radiat Oncol Biol Phys 9:633-642, 1983

9. DeLaney TF, Kepka L, Goldberg SI, Hornicek FJ, Gebhardt MC, Yoon SS, et al: Radiation therapy for control of softtissue sarcomas resected with positive margins. Int J Radiat Oncol Biol Phys 67:1460-1469, 2007

10. DeLaney TF, Liebsch NJ, Pedlow FX, Adams J, Weyman EA, Yeap BY, et al: Long-term results of Phase II study of high dose photon/proton radiotherapy in the management of spine chordomas, chondrosarcomas, and other sarcomas. J Surg Oncol 110:115-122, 2014

11. Holliday EB, Mitra HS, Somerson JS, Rhines LD, Mahajan A, Brown PD, et al: Postoperative proton therapy for chordomas and chondrosarcomas of the spine: adjuvant versus salvage radiation therapy. Spine (Phila Pa 1976) 40:544-549, 2015

12. Indelicato DJ, Rotondo RL, Begosh-Mayne D, Scarborough MT, Gibbs CP, Morris CG, et al: A prospective outcomes study of proton therapy for chordomas and chondrosarcomas of the spine. Int J Radiat Oncol Biol Phys 95:297-303, 2016

13. Keisch ME, Garcia DM, Shibuya RB: Retrospective longterm follow-up analysis in 21 patients with chordomas of various sites treated at a single institution. J Neurosurg 75:374-377, 1991

14. Lomax A: Intensity modulation methods for proton radiotherapy. Phys Med Biol 44:185-205, 1999
15. McDonald MW, Linton OR, Moore MG, Ting JY, CohenGadol AA, Shah MV: Influence of residual tumor volume and radiation dose coverage in outcomes for clival chordoma. Int J Radiat Oncol Biol Phys 95:304-311, 2016

16. Molina CA, Ames CP, Chou D, Rhines LD, Hsieh PC, Zadnik PL, et al: Outcomes following attempted en bloc resection of cervical chordomas in the $\mathrm{C}-1$ and $\mathrm{C}-2$ region versus the subaxial region: a multiinstitutional experience. J Neurosurg Spine 21:348-356, 2014

17. Pennicooke B, Laufer I, Sahgal A, Varga PP, Gokaslan ZL, Bilsky MH, et al: Safety and local control of radiation therapy for chordoma of the spine and sacrum: a systematic review. Spine (Phila Pa 1976) 41 (Suppl 20):S186-S192, 2016

18. Potluri S, Jefferies SJ, Jena R, Harris F, Burton KE, Prevost AT, et al: Residual postoperative tumour volume predicts outcome after high-dose radiotherapy for chordoma and chondrosarcoma of the skull base and spine. Clin Oncol (R Coll Radiol) 23:199-208, 2011

19. Rich TA, Schiller A, Suit HD, Mankin HJ: Clinical and pathologic review of 48 cases of chordoma. Cancer 56:182187,1985

20. Rotondo RL, Folkert W, Liebsch NJ, Chen YL, Pedlow FX, Schwab JH, et al: High-dose proton-based radiation therapy in the management of spine chordomas: outcomes and clinicopathological prognostic factors. J Neurosurg Spine 23:788-797, 2015

21. Rutz HP, Weber DC, Sugahara S, Timmermann B, Lomax AJ, Bolsi A, et al: Extracranial chordoma: outcome in patients treated with function-preserving surgery followed by spot-scanning proton beam irradiation. Int J Radiat Oncol Biol Phys 67:512-520, 2007

22. Schwab JH, Healey JH, Rose P, Casas-Ganem J, Boland PJ: The surgical management of sacral chordomas. Spine (Phila Pa 1976) 34:2700-2704, 2009

23. Snider JW, Schneider RA, Poelma-Tap D, Stieb S, Murray FR, Placidi L, et al: Long-term outcomes and prognostic factors after pencil-beam scanning proton radiation therapy for spinal chordomas: a large, single-institution cohort. Int J Radiat Oncol Biol Phys 101:226-233, 2018

24. Stieb S, Snider JW III, Placidi L, Kliebsch U, Lomax AJ, Schneider RA, et al: Long-term clinical safety of high-dose proton radiation therapy delivered with pencil beam scanning technique for extracranial chordomas and chondrosarcomas in adult patients: clinical evidence of spinal cord tolerance. Int J Radiat Oncol Biol Phys 100:218-225, 2018

25. Talac R, Yaszemski MJ, Currier BL, Fuchs B, Dekutoski $\mathrm{MB}, \mathrm{Kim} \mathrm{CW}$, et al: Relationship between surgical margins and local recurrence in sarcomas of the spine. Clin Orthop Relat Res (397):127-132, 2002

26. Verlaan JJ, Kuperus JS, Slooff WB, Hennipman A, Oner FC: Complications, secondary interventions and long term morbidity after en bloc sacrectomy. Eur Spine J 24:2209_ 2219, 2015

27. Weber DC, Malyapa R, Albertini F, Bolsi A, Kliebsch U, Walser M, et al: Long term outcomes of patients with skullbase low-grade chondrosarcoma and chordoma patients treated with pencil beam scanning proton therapy. Radiother Oncol 120:169-174, 2016

28. Weber DC, Murray F, Combescure C, Calugaru V, Alapetite C, Albertini F, et al: Long term outcome of skull-base chondrosarcoma patients treated with high-dose proton therapy with or without conventional radiation therapy. Radiother Oncol 129:520-526, 2018

29. Wei F, Liu Z, Liu X, Jiang L, Dang G, Passias PG, et al: An approach to primary tumors of the upper cervical spine with spondylectomy using a combined approach: our experience with 19 cases. Spine (Phila Pa 1976) 43:81-88, 2018

30. York JE, Berk RH, Fuller GN, Rao JS, Abi-Said D, Wildrick 
DM, et al: Chondrosarcoma of the spine: 1954 to 1997. J Neurosurg 90 (1 Suppl):73-78, 1999

31. Zagars GK, Ballo MT: Significance of dose in postoperative radiotherapy for soft tissue sarcoma. Int J Radiat Oncol Biol Phys 56:473-481, 2003

\section{Disclosures}

Dr. Snider: consultant for Siemens Healthineers, Pyrexar, and Varian Medical Systems; and patent holder with ProtonGRID.

\section{Author Contributions}

Conception and design: Weber. Acquisition of data: Murray,
Snider. Analysis and interpretation of data: Weber, Murray. Drafting the article: Murray. Critically revising the article: Weber, Snider, Schneider, Walser, Bolsi, Pica, Lomax. Reviewed submitted version of manuscript: Weber, Snider, Schneider, Walser, Bolsi, Pica, Lomax. Approved the final version of the manuscript on behalf of all authors: Weber. Statistical analysis: Murray, Snider. Study supervision: Weber.

\section{Correspondence}

Damien C. Weber: Center for Proton Therapy, Paul Scherrer Institute, ETH Domain, Villigen, Switzerland. damien.weber@psi. ch; damiencharles.weber@uzh.ch. 